\title{
Trastorno Específico del Lenguaje: Una revisión
}

\author{
Specific Language Impairment: \\ A Review
}

Douglas B. Petersen Division of Communication Disorders College of Health Sciences University of Wyoming

Catherine M. Gardner Behavioral Consultation Services of Northern Arizona

\section{RESUMEN}

El Trastorno Específico del Lenguaje (TEL) es uno de los trastornos infantiles más comunes y afecta aproximadamente al $7 \%$ de los niños en los Estados Unidos de Norteamérica. Si bien se trata de un trastorno prevalente, su origen no se ha esclarecido del todo y existen diversas teorías al respecto. El propósito de esta revisión es presentar un panorama global de los enfoques teóricos que pretenden explicar el TEL y ofrecer una mirada crítica a las investigaciones que apoyan estas teorías. Una explicación sencilla para el origen de este déficit podría relacionarse con la existencia de un correlato neural específico deficitario para cada subgrupo de TEL descrito. Dado que falta evidencia consistente al respecto, una definición amplia del TEL considerando todos los subgrupos queda sin verificación empírica todavía. Sin embargo, los estudios siguen progresando hacia esta dirección ofreciendo evidencias para apoyar o rechazar las diversas teorías acerca del TEL.

Palabras clave: Trastorno Específico del Lenguaje, teorías explicativas del Trastorno Específico del Lenguaje.

\section{ABSTRACT}

The Specific Language Impairment (SLI) is one of the most common childhood disorders, affecting an estimated $7 \%$ of children in the United States of America. Although SLI is a prevalent disorder, it is not well understood, and a number of theories associated with the explanation of specific language impairment have been presented. The purpose of this review is to present an overview of theories of specific language impairment and critically discuss the research supporting those theories. It may be that the most parsimonious account for $S \mathrm{LI}$ is that there are different subgroups of $\mathrm{SLI}$ and that there is a direct correlation between the specific neural damage and the corresponding subgroup. A broad definition of SLI with accompanying sub-categories remains to be empirically verified. Nonetheless, research is progressing in that direction. It is clear that additional research is needed to support or refute the varying theories of SLI.

Keywords: Specific Language Impairment, theoretical approaches of SLI. 


\section{Introducción}

Los profesionales que trabajan con niños que tienen dificultades de lenguaje se encuentran con frecuencia con un tipo de déficit conocido como Trastorno Específico del Lenguaje (TEL). Los niños con TEL presentan deficiencias significativas en su desempeño lingüístico sin que se asocien a alteraciones neurológicas evidentes, pérdidas auditivas, carencias ambientales extremas o baja inteligencia no-verbal ${ }^{1}$.

Los datos acerca de la prevalencia del TEL lo señala como uno de los trastornos más comunes en la infancia, afectando aproximadamente al $7 \%$ de la población infantil norteamericana ${ }^{2}$. Sin embargo, a pesar de su alta frecuencia, su etiología aún no encuentra una explicación satisfactoria entre los investigadores, lo que ha generado diversas teorías al respecto.

Los diversos planteamientos sobre el origen del TEL se han agrupado en dos enfoques principales: el lingüístico y el de procesamiento.

El primero se inscribe básicamente en la teoría que plantea un déficit selectivo en los módulos especializados para el aprendizaje del lenguaje. Estas explicaciones sugieren que los errores morfo-sintácticos que presentan los niños con TEL se deben a un conocimiento limitado de las reglas gramaticales del lenguaje. Se considera el problema específico del dominio y relacionado con un módulo cerebral que procesa la morfosintaxis ${ }^{3,4}$.
En contraste con las teorías lingüísticas, el enfoque de procesamiento con respecto al TEL no se enfoca exclusivamente en las deficiencias lingüísticas, por lo que no propone necesariamente la existencia de un módulo específico del cerebro que regule la adquisición gramatical. Las explicaciones del déficit de procesamiento en el TEL sugieren más bien un déficit en la habilidad cerebral para interpretar el input lingüístico y/o acceder al conocimiento lingüístico almacenado en la memoria ${ }^{5,6}$. Por lo general, las explicaciones desde este enfoque plantean que existe un déficit de funcionamiento en la memoria de trabajo fonológica o una deficiencia en la velocidad de procesamiento de la información verbal'.

El propósito de este artículo es presentar un análisis actualizado de las diferentes posturas y teorías acerca del origen y naturaleza del TEL.

\section{El enfoque lingüístico}

\section{La Explicación del Infinitivo Opcional Extendido}

El planteamiento acerca del infinitivo opcional propuesto por Rice y cols. ${ }^{8}$ es la explicación lingüística más prominente acerca de las dificultades morfosintácticas que evidencian los niños con TEL hablantes del inglés. Esta teoría sugiere que, alrededor de los tres años, los niños con desarrollo típico del lenguaje realizan producciones verbales usando el infinitivo opcional, cuando las formas finitas de los verbos en inglés son opcionales"-11 (ej. "daddy swing/ daddy swings"). Con frecuencia se ha observado 
que los niños con TEL mantienen el uso del infinitivo opcional (revelando sus dificultades con los morfemas gramaticales) en edades en las que la mayor parte de los niños con desarrollo típico ya dominan las obligaciones morfológicas del lenguaje ${ }^{12}$. La explicación del infinitivo opcional sugiere que los niños con TEL no reconocen la naturaleza obligatoria de la morfología temporal de los verbos en inglés y consideran el uso de los infinitivos como 'opcional'13.

Rice y sus colegas han comprobado la explicación del infinitivo opcional a partir de diversas investigaciones experimentales. Por ejemplo, Rice et al. ${ }^{8}$, Rice y Wexler ${ }^{13}$, y Cleave y Rice ${ }^{14}$, investigaron si los niños con TEL hablantes del inglés tenían más dificultad con la morfología temporal o con la morfología no-temporal al compararlos con sus pares con desarrollo típico por edad y por desarrollo de lenguaje (mismo promedio de morfemas por frase). Los resultados evidenciaron que los niños con TEL tenían mayor dificultad con la morfología temporal en relación a los otros dos grupos. Específicamente en el presente en tercera persona singular "s" (ej. David swings); en el tiempo pasado regular "ed" (ej. plaved), en el verbo auxiliar "do" (ej. she did) y en la cópula/auxiliar "be" (ej. she is ). Paralelamente los niños con TEL no evidenciaron dificultades inusuales en la morfología no temporal de verbos en inglés, tal es el caso del morfema "s" para el plural regular (ej. three dogs $)$; las preposiciones "in" y "on", ni el progresivo - "ing” (ej. talking).

Cleave y Rice ${ }^{14}$ estudiaron el uso de la cópula verbal contraída y no contraída con el verbo auxiliar "ser" (en un ejemplo del inglés: "He is" y He's). Ellos encontraron que los niños con TEL producen más errores con la cópula no contraída al mismo tiempo que utilizan la forma contraída con una frecuencia similar a la de los niños con desarrollo típico del lenguaje.

En diversas investigaciones efectuadas para probar el planteamiento del infinitivo opcional para el TEL se seleccionaron los niños con criterios de inclusión similares a los utilizados en el proyecto de Rice y Wexler ${ }^{13}$. Lo anterior es problemático, porque tales criterios no fueron del todo estandarizados y es posible que no fueran suficientes. Por ejemplo, se reclutaron niños con TEL diagnosticados por fonoaudiólogos en el proyecto anterior de Rice et $a 1 .^{8}$, quienes no siempre utilizaron los mismos instrumentos de evaluación por lo que el diagnóstico de trastorno de lenguaje dependía además del juicio de cada profesional. Así, dos de los niños que participaron en el proyecto de Rice y Wexler ${ }^{13}$ recibieron puntajes dentro de una desviación estándar bajo el promedio en el Test of Language DevelopmentPrimary ${ }^{15}$ (TOLD:P-3) y sin embargo, se incluyeron en el grupo experimental de TEL. Además, no se incluían los niños con problemas severos de articulación.

Otro aspecto que puede criticarse, fuera de los criterios de inclusión de los participantes, es el uso de un diseño experimental de grupo, tal como lo emplearon estos investigadores. De este modo, un análisis de los resultados del proyecto de Rice y Wexler ${ }^{13}$ reveló que los errores de tan solo unos cuantos sujetos del grupo con TEL modificaron 
el promedio del grupo experimental. En otras palabras, es posible que sólo unos pocos niños del grupo con TEL hayan tenido dificultades inusuales con la morfología relacionada con el tiempo verbal y que otros no las tuvieran. El estudio de Rice y Wexler ${ }^{13}$ reveló que algunos de los niños en el grupo experimental de Trastorno Específico del Lenguaje produjeron morfemas temporales por encima del promedio de los dos grupos de control. Es posible que estos hallazgos indiquen que sólo un subgrupo de los niños con Trastorno Específico del Lenguaje presentaba dificultades con la morfología temporal. Esto sugeriría que es posible usar este tipo de tareas como un eventual marcador clínico de alta especificidad pero baja sensibilidad.

Otros estudios de Rice y su equipo ${ }^{16-21}$, han trabajado sobre la misma muestra de la investigación de Rice y Wexler ${ }^{13}$ para el estudio de factores relacionados con el uso extendido del infinitivo opcional. De modo similar, en otras investigaciones realizadas por estos investigadores se han mantenido criterios similares de inclusión para los grupos experimentales de niños con $\mathrm{TEL}^{8,14,22,23}$. La amplitud en los criterios de inclusión no considera la posibilidad de que sólo un subgrupo de niños con TEL puede manifestar este tipo de errores en la morfología del pasado verbal.

\section{Trastorno Específico del Lenguaje Gramatical}

Debido a que es posible que el TEL sea un trastorno heterogéneo en el cual solo un porcentaje de niños tiene problemas con la morfología temporal, Van der Lely y otros investigadores han intentado enfocar sus estudios específicamente en un subgrupo relativamente homogéneo, los niños con Trastorno Específico del LenguajeGramatical $^{24}$ (TEL-G) que afectaría entre el 10 y $20 \%$ de los niños con TEL ${ }^{25}$. Ellos presentarían una deficiencia primaria y desproporcionada en el sistema de computación de la gramática ${ }^{24,26-29}$. Esta explicación, parecida a la del infinitivo opcional, se sustenta en la especificidad funcional del cerebro para el procesamiento gramatical, función que podría afectarse de manera diferencial ${ }^{30-33}$.

En el estudio de los niños con TEL-G, algunos investigadores como Van der Lely ${ }^{25,27}$ han empleado criterios más exigentes para la inclusión de los participantes que los descritos en el proyecto de Rice y Wexler ${ }^{13}$. Además de los criterios 'estándar' para efectuar el diagnóstico de este cuadro, los niños que presentan TEL- Gramatical, no pueden evidenciar problemas simultáneos de la producción del habla, deficiencias pragmáticas o impedimentos no-verbales ${ }^{34}$.

Van der Lely y su equipo aparentemente han identificado un subgrupo de niños que presentan TEL- Gramatical. Dado que la prevalencia estimada para este trastorno es precisa (alrededor del 10 a 20\% de los niños son clasificados tradicionalmente con TEL), no es de sorprender que Rice y cols. ${ }^{13}$ en su estudio randomizado de casos y controles, usaran medias y desviaciones estándares como procedimiento primario de análisis, encontrando diferencias significativas con sólo algunos de los morfemas testeados. El uso extendido del infinitivo opcional implicaría una dificultad sólo con la morfología 
relacionada con el pasado; en ese sentido la investigación conducida por Rice ${ }^{13}$ sustenta esta hipótesis. Sin embargo, es posible que sólo esos niños con TEL-G, incluidos en el estudio señalado previamente, tengan dificultades suficientes en los morfemas relacionados con la temporalidad, como para influir significativamente en el promedio del grupo comparado con el promedio del grupo control. Al mismo tiempo, es posible que esos niños con TEL-G tengan, de igual manera, algunas dificultades con partículas morfológicas que no se relacionan con la temporalidad, pero con insuficiente fuerza como para influir significativamente al $80-90 \%$ de los niños restantes de la muestra con TEL (pero que no tenían déficits morfológicos significativos).

Hay poca controversia con respecto a que existe un porcentaje de los niños que hablan inglés con TEL que tienen dificultades con la morfología temporal. Existe aún menos discusión en cuanto a si estos niños tienen problemas con la morfología gramatical en general. En este sentido, distintos investigadores han encontrado que los niños con TEL tienden a usar los morfemas gramaticales en menor proporción que los niños más jóvenes con los mismos promedios de morfemas por frase en contextos obligatorios ${ }^{35-40}$. Sin embargo, sí existe mucho debate con respecto a por qué tal dificultad gramatical existe. Así, se han propuesto explicaciones alternativas, fuera de la hipótesis del infinitivo opcional.

\section{El enfoque de procesamiento}

Estos postulados abordan por una parte, hipótesis orientadas al procesamiento de información general y por otro lado explicaciones acerca de un déficit de procesamiento más específico que apuntan a tres posibles aspectos deficitarios: a) déficits de velocidad para procesar información, b) dificultades en la representación fonológica, y c) problemas en la percepción auditiva ${ }^{41,42}$.

\section{La explicación sobre el procesamiento de información general}

Este postulado sugiere que los niños con TEL tienen una capacidad disminuida para procesar tanto la información lingüística como la no lingüística. El procesamiento limitado consistiría en una restricción en la capacidad de la memoria de trabajo, una disminución en la cantidad de energía necesaria para procesar la información, o una combinación de ambas ${ }^{1,43-46}$.

Se han estudiado el recuerdo y la búsqueda de palabras ${ }^{47-49}$; la semántica ${ }^{41,50,51}$; el reconocimiento táctil ${ }^{52-55}$; la inferencia ${ }^{56,57}$; y muchas otras destrezas lingüísticas y no lingüísticas en los niños con TEL. En todos los estudios se ha concluido que cierto grado de déficit de procesamiento general sería el responsable de las deficiencias en los grupos estudiados.

Hayiou-Thomas, Bishop y Plunkett $^{58}$ intentaron provocar conductas similares a las que aparecen en los niños con TEL en un grupo de niños con desarrollo típico, al someterlos a factores 
estresantes, cognitivos y de memoria, mientras realizaban una tarea de juzgar gramaticalidad. Los investigadores señalaron que cuando la señal de habla se presentaba a una velocidad normal, el desempeño fue excelente. Sin embargo, cuando se aceleró la señal al doble de la velocidad normal (supuestamente para simular una deficiencia de procesamiento), los niños con desarrollo típico cometieron errores morfo-sintácticos que se suelen indicar en los estudios relativos al infinitivo opcional extendido. Es decir, se produjeron de manera incorrecta los verbos en tiempo pasado y la 's' de la tercera persona singulary correctamente la 's' no-temporal. El hallazgo de que un aumento en las demandas de procesamiento y la memoria desencadenó conductas parecidas a las de los niños con TEL- Gramatical en los niños con desarrollo típico, sustentaría la explicación de un déficit de procesamiento general para el TEL.

Bajo este supuesto, es posible explicar una variedad más amplia de dificultades de lenguaje en comparación con una teoría más restringida como la explicación del infinitivo opcional extendido y sustentar con mayor solidez la heterogeneidad de rasgos de la población con TEL, pero también se le ha criticado por ser demasiado general ${ }^{41,59,60}$. Como respuesta a estas críticas, los investigadores se han enfocado al estudio de habilidades de procesamiento más específicas.

\section{La hipótesis superficial}

Leonard $^{61}$, Leonard, McGregor \& Allen ${ }^{37}$, y Leonard, Eyer, Bedore \& Grela ${ }^{39}$, sustentan la hipótesis superficial. Este planteamiento concuerda con la explicación de deficiencia en el procesamiento de información general con un énfasis más específico en la rapidez. Propone que los niños con TEL tienen una habilidad de procesamiento general limitada y que ésta tiene un efecto adverso sobre la adquisición de morfemas que son menos sobresalientes fonéticamente y de corta duración. Este planteamiento no supone una dificultad en percibir los morfemas gramaticales, sino que los morfemas gramaticales requieren mayor demanda cognitiva, lo que debilitaría un sistema de procesamiento ya deficitario. Debido a que el habla típica es rápida y producida en tiempo real, los niños con TEL no pueden procesar adecuadamente los diversos morfemas breves; así, bajo esta premisa, tendrían mayor dificultad tanto en los morfemas gramaticales relacionados con la temporalidad como con aquellos que no lo están.

Montgomery y Leonard ${ }^{62}$ establecieron criterios de selección para los participantes de sus investigaciones similares a los de Van der Lely ${ }^{63} \mathrm{y}$ Van der Lely \& Stollwerck ${ }^{25,29}$. Consideraron un desempeño bajo una desviación estándar de la media en el Test of Reception of Grammar (TROG; Bishop ${ }^{64}$ ) para que los niños con TEL fueran asignados al grupo experimental, asegurándose así que presentaban un déficit en la gramática receptiva. Compararon el procesamiento de morfemas con mayor y menor substancia fonética en 21 niños con TEL con sus pareos por edad y desempeño en la sintaxis receptiva. Los hallazgos indican que los niños con desarrollo típico rinden mejor que los niños con TEL con respecto al tiempo de reacción y juicio de gramaticalidad de las inflexiones de poca substancia fonética. Además, los investigadores encontraron que los 
errores de morfología gramatical (temporales y no temporales) fueron significativamente más en los niños con TEL que en sus pares de la misma edad.

La explicación de la hipótesis superficial presentada por Leonard y sus colegas también ha encontrado apoyo en investigaciones translingüísticas recientes. Leonard y Bortolini ${ }^{65}$ investigaron niños hablantes nativos del italiano con TEL evidenciando que ellos producen el tiempo verbal de la tercera persona singular tan correctamente como sus pares sin dificultades de lenguaje, contradiciendo los hallazgos de los estudios en inglés con respecto a la hipótesis del infinitivo opcional. De hecho, una síntesis de las investigaciones trans-lingüísticas evidencia diferencias en cuanto al desempeño en morfología en los diferentes idiomas ${ }^{1,39,66-71}$.

Es interesante comentar que los niños con TEL que están adquiriendo una lengua con una morfología inflexional bastante evidente, como el italiano o el español, pueden producir estos constituyentes morfológicos con mayor consistencia que los que adquieren un idioma con una morfología inflexional más débil, como el inglés. Los hallazgos principales de estos proyectos ponen de manifiesto que algunas morfologías gramaticales pueden ser un problema más o menos relevante para los niños con TEL según la lengua que adquieran. Lo anterior sugiere que las dificultades gramaticales no se deberían a problemas con un módulo particular del cerebro, sino que a déficits en identificar e interpretar el insumo lingüístico ${ }^{72}$.

\section{La teoría del déficit de la percepción auditiva}

Muchos investigadores han postulado que los niños con TEL tienen dificultad para percibir y procesar los estímulos auditivos ${ }^{73-76}$. Un proyecto reciente de Burlingame, Sussman, Gillam y $\mathrm{Hay}^{77}$, encontró que los niños con TEL fueron menos consistentes en la identificación de habla sintética cuando variaba en la duración de formantes de transición (/ba/ a /wa/ y /da/ a /ja/). $\mathrm{Al}$ respecto, los autores sugieren una deficiencia en el procesamiento de los sonidos del habla. De igual manera, Corriveau, Pasquini, y Goswami ${ }^{78}$ encontraron que de 70 a $80 \%$ de los niños con TEL estudiados tenían problemas de percepción auditiva. Ziegler, Pech-George, George, Alario y Lorenzi ${ }^{79}$, investigaron las habilidades de percepción auditiva de niños con TEL y con desarrollo típico del lenguaje, presentándoles estímulos auditivos en presencia de sonidos enmascarantes. Los niños con TEL repitieron las consonantes con mayor dificultad que los del grupo control. Ante estos resultados, los autores propusieron que los niños con TEL tenían un déficit en la percepción (auditiva) del habla.

\section{Deficiencias de la memoria fonológica}

A pesar de los hallazgos descritos, es complejo determinar si los niños con TEL tienen dificultad en la percepción auditiva o en la memoria y el procesamiento. Gathercole y Baddeley ${ }^{80,81}$ han revelado que estos niños pueden tener problemas con la memoria de trabajo fonológica debido a la dificultad relativa para repetir pseudopalabras y recordar una lista de palabras, cuando se les compara con niños de desarrollo típico. Según sus 
resultados, no hubo diferencias significativas entre ambos grupos al discriminar fonemas diferentes ni tampoco con la velocidad de articulación. Debido a esto, los investigadores proponen que la dificultad mostrada por estos niños podría relacionarse con una limitación en la memoria de trabajo fonológica.

Si no se consideran la memoria de trabajo fonológica u otros factores de procesamiento durante la evaluación de las destrezas de percepción auditiva de niños con TEL, resulta imposible determinar la causa del déficit. Por lo tanto, pudiera ser que los niños con TEL que presenten dificultades para repetir y recordar los estímulos auditivos, tengan un déficit en la percepción auditiva, de la memoria de trabajo fonológica, ambas, o eventualmente en ninguna de ellas. Reconociendo la complejidad para establecer evidencias claras en relación a uno u otro aspecto, Gathercole y Baddeley ${ }^{80,81}$ encontraron que cuando disminuyeron las demandas de la memoria, los niños con TEL percibieron los sonidos del habla tan bien como sus pares sin TEL de la misma edad. Esto sugiere que eventualmente los niños con TEL no tendrían problemas en la percepción auditiva, sino en la memoria. En esa misma línea de evidencia, Sussman ${ }^{82}$ reportó que los niños con TEL pudieron discriminar entre los estímulos auditivos [ba] y [da] con una precisión parecida a la de niños de desarrollo típico pareados por edad y también por desarrollo del lenguaje. Los niños con TEL tuvieron mayor dificultad de precisión en estímulos presentados al final. Por tanto, Sussman ${ }^{82}$ llegó a hipotetizar que el problema se debía a una dificultad para mantener la información en la memoria de trabajo fonológica.

Otros investigadores también han atribuido el TEL a un problema específico de la memoria de trabajo fonológica ${ }^{83-86}$. Gillam et al. ${ }^{83}$ seleccionaron niños con TEL y controles pareados por edad, nivel de lectura y habilidad para recordar números. La tarea solicitada consistió en recordar una serie de números incluyendo o no una palabra sin sentido en posición final de la lista; se les instruyó además en ignorar la palabra cuando apareciera. Los niños con TEL tuvieron bastante más dificultad en recordar los últimos números de la lista cuando se incluía la pseudopalabra. Ante esta evidencia, los autores postularon que estos niños procesaron más lentamente la información fonológica en la memoria de trabajo fonológica.

En un proyecto relacionado, Gillam y su grupo $^{42}$ probaron distintas hipótesis controvertidas relacionadas con el procesamiento de información en los niños con TEL. Les solicitaron a los niños con TEL y sus respectivos controles recontar una serie de números presentados visual, auditiva o audiovisualmente. Los niños respondieron repitiendo los números verbalmente o señalándolos en una pantalla de computador. Los resultados indicaron que los niños con TEL mostraron peor desempeño bajo la modalidad visual. Según los autores, estos resultados dan cuenta del compromiso de la memoria de trabajo fonológica. El diseño del proyecto permitió algún grado de confianza al interpretar que las deficiencias de los niños no se relacionaban con la producción del habla 
(los niños con TEL rendían mejor cuando se requería la tarea de producción verbal), ni con el procesamiento auditivo (se observó mejor desempeño cuando había señal auditiva) o con la velocidad con que se presentaban los estímulos fonológicos (una presentación más lenta fue la condición más difícil para ambos grupos). Gillam et $\mathrm{al}^{42}$ concluyeron que las deficiencias de la memoria de trabajo fonológica en los niños con TEL se debían a la dificultad en transformar y retener las representaciones fonológicas mentales más específicas y que el procesamiento mental adicional requerido por la tarea sobreexigió su memoria de trabajo fonológica. Los investigadores hicieron notar que se había establecido una tasa cómoda y condiciones óptimas en la presentación de los estímulos auditivos (i.e. audífonos), evitando así un factor que puede confundir la influencia del procesamiento auditivo como un factor contribuyente. Sin embargo, el hallazgo de que los niños con TEL tuvieron mayor dificultad con la tarea cuando no se presentó un estímulo auditivo sugiere un problema de la memoria de trabajo fonológica.

Aunque existe bastante evidencia para apoyar la idea de que la memoria de trabajo fonológica es un factor contribuyente al TEL, lo más probable es que sirva sólo como explicación parcial.

Existen otras deficiencias documentadas que no dicen relación con la memoria de trabajo fonológica, incluyendo a la memoria visual, así como también otros déficits no lingüísticos ${ }^{87,88}$.

\section{La hipótesis del déficit procedural}

Rice y Wexler ${ }^{13}$ sugieren que una cuestión crítica es la identificación de un marcador clínico: una forma lingüística o un principio que se puede comprobar como característico del Trastorno Específico del Lenguaje. Sin embargo, la manifestación heterogénea del cuadro en diferentes idiomas, dificulta la tarea de identificar un solo marcador, como por ejemplo, el manejo deficitario del marcador morfológico de tiempo.

Con la variabilidad aparente entre los niños con Trastorno Específico del Lenguaje, no es de sorprender que existan tantas teorías y que cada una tenga algunas evidencias. Así, otra teoría explicativa posible es la relativa al déficit procedural planteada por Ullman y Pierpont ${ }^{89}$, quienes plantearon lo siguiente:

"Although previously proposed explanatory hypotheses can individually capture specific aspects of the empirical data, none of them can easily account for either the range or the variation of the particular impaired linguistic and nonlinguistic functions found across SLI, and even within SLI subgroups. Indeed, we believe that any purely functional account of SLI will have difficulty explaining the variety of impairments that occur even within individuals with SLI".

Estos investigadores sugieren que un análisis de los rasgos neurológicos puede proveer una buena estrategia para entender el TEL. Desde su perspectiva, habría pocas hipótesis que intentan relacionar el funcionamiento cerebral con los impedimentos cognitivos evidenciados 
en los niños con TEL, o explicar el rango de anormalidades observadas en el trastorno. Para responder a esta limitación teórica, han presentado la hipótesis de deficiencias de procedimiento. Existiría un número considerable de individuos con TEL que evidencian anomalías cerebrales asociadas principalmente a los circuitos del lóbulo frontal y los ganglios basales, estructuras que constituyen el sistema de la memoria de procedimiento. Tales anormalidades dentro del sistema de la memoria de procedimiento (compuesto por estructuras constituyentes diferentes) podrían potencialmente explicarlas varias manifestaciones del Trastorno Específico del Lenguaje y asociarse a la heterogeneidad de conducta.

Los sistemas mnésicos del cerebro incluyen los sistemas de la memoria declarativa y de procedimiento. La memoria declarativa rige sobre el léxico, mientras que el conocimiento específico lo hace sobre las palabras y la información irregular o no predecible. La memoria de procedimiento está asociada a los registros gobernados por reglas: la sintaxis, la morfología y la fonología. Este sistema se ha ligado directamente a la memoria de trabajo fonológica, el procesamiento temporal (por ejemplo, la percepción de los estímulos presentados rápidamente, el ritmo, velocidad), la sintaxis no lexicalizada, y la morfología determinada por reglas, como el tiempo verbal pasado regular 'ed' y la fonología (como representaciones fonológicas nuevas).

El sistema de la memoria de procedimiento también se ha vinculado indirectamente a la memoria léxica/declarativa, sobre todo cuando se tiene que aprender el vocabulario por medio de la inferencia, cuando queda gran cantidad de información en la memoria de trabajo fonológica o cuando se presenta la información rápidamente. También se la asocia al aprendizaje de palabras (cuando se presentan los estímulos rápidamente y $\sin$ contexto), a los impedimentos motores como formar una secuencia, el habla, "timming" y el equilibrio, así como a las imágenes mentales dinámicas.

Lahipótesis de deficiencias de procedimiento permite establecer una clasificación acerca de un trastorno procedural para el lenguaje, proponiendo que "muchos, si no la mayoría de los individuos con TEL, tienen anormalidades con el sistema procedimental del cerebro, las cuales resultan en impedimentos gramaticales $\mathrm{y} / \mathrm{o}$ deficiencias en acceso al léxico" ${ }^{19}$. La hipótesis de deficiencias de procedimiento toma en cuenta los problemas no lingüísticos al sugerir que éstos pueden ocurrir debido a la interdependencia del sistema de procedimiento para funcionar.

\section{Intervención}

Puede ser que la explicación más sencilla para el TEL es que existen diversos subgrupos y que existiría una correlación directa entre los daños neurales específicos y el subgrupo correspondiente.

Una definición amplia del TEL que incluya los distintos subtipos queda sin verificación empírica todavía. Sin embargo, los estudios siguen progresando hacia esta dirección. E1 rótulo diagnóstico de TEL puede ser apropiado 
para niños con dificultades relacionadas a la morfología de los tiempos verbales, limitaciones generalizadas de la morfología, o impedimentos del procesamiento lingüístico sin problemas significativos de morfología. Por otra parte, esa misma nomenclatura resulta como diagnóstico aceptable si se identifica un trastorno neurológico manifestado como un impedimento de lenguaje y se puede eliminar como causa un problema auditivo, coeficiente intelectual no-verbal bajo, daño neurológico evidente o factores ambientales. Es decir, si es posible reunir los criterios de exclusión, se puede considerar el Trastorno Específico del Lenguaje como un diagnóstico amplio, tal como el cáncer o el Trastorno del Desarrollo Pervasivo, que requieren sub-clasificaciones específicas. Igualmente, la clasificación del Trastorno Específico del Lenguaje podría beneficiarse de subtipologías clínicas.

Está claro que hacen falta investigaciones adicionales para apoyar o rechazar las diversas teorías acerca del origen del TEL; de igual manera, los resultados del tratamiento basado en una teoría en particular deben aportar evidencia que sustente o disminuya el apoyo para tal teoría, permitiendo acercarse a una explicación comprensiva del cuadro.

\section{Referencias}

1. Leonard, L. (1998) Children with specific language impairment. Cambridge, MA: MIT Press.

2. Tomblin, J.B.; Smith, E. \& Zhang, X. (1997) Epidemiology of specific language impairment: Prenatal and perinatal risk factors. J Commun Disord, 30, 325-344.
3. Kelley, E.; Jones, G. \& Fein, D. (2003) Language assessment in children. En M. Hersen, \& S.R. Beers (Eds.) Comprehensive Handbook of Psychological Assessment, Volume 1: Intellectual and Neuropsychological Assessment. New York, NY: John Wiley \& Sons, Inc. pp. 191-215

4. Van der Lely, H.K.J. (2005) Grammatical-specific language impairment and the computational grammatical complexity hypothesis. Revue Frequences, 17(3), 13-20.

5. Ellis-Weismer, S.E.; Evans, J. \& Hesketh, L.J. (1999) An examination of verbal working memory capacity in children with specific language impairment. J Speech Lang Hear $R$, 42, 1249-1260.

6. Windsor, J.; Milbrath, R.L.; Carney, E.J. \& Rakowski, S.E. (2001) General slowing in language impairment: Methodological considerations in testing the hypothesis. J Speech Lang Hear R, 44, 446-461.

7. Montgomery, J.W. \& Windsor, J. (2007) Examining the language performances of children with and without specific language impairment: Contributions of phonological short-term memory and speed of processing. J Speech Lang Hear R, 50, 778-797.

8. Rice, M.L.; Wexler, K. \& Cleave, P. (1995) Specific language impairment as a period of extended optional infinitive. J Speech Lang Hear R, 38, 850-863.

9. Weverink, M. (1990) What's missing in Dutch? PRCLD, 29, 125-132.

10. Pierce, A. (1992) Language acquisition and syntactic theory. Dordrecht, The Netherlands: Kluwer.

11. Wexler, K. (1994) Optional infinitives, head movement and the economy of derivations in child grammar. In D. Lightfoot \& N. Hornstein (Eds.) Verb movement. Cambridge, MA: Cambridge University Press, pp. 305350 .

12. Brown, M. (2004) Optional infinitives in Down syndrome. Massachusetts Institute of Technology: Abnormal Language, 9.56J/24.507J.

13. Rice, M. \& Wexler, K. (1996) Toward tense as a clinical marker of specific language impairment in English speaking children. J Speech Hear Res, 39, 1239-1257.

14. Cleave, P.L. \& Rice, M.L. (1997) An examination of the morpheme BE in children with specific language impairment: The role of contractibility and grammatical form class. J Speech Lang Hear R, 40, 480-492. 
15. Newcomer, P. \& Hammill, D. (1997). Test of language development-Primary: 3. Austin, TX: Pro-Ed.

16. Redmond, S.M. \& Rice, M.L. (1998) The socioemotional behaviors of children with specific language impairment: Social adaptation or social deviance? J Speech Lang Hear R, 41, 688-700.

17. Redmond, S.M. \& Rice M.L. (2001) Detection of irregular verb violations by children with and without specific language impairment. J Speech Lang Hear R, 44, 655-669.

18. Redmond, S.M. \& Rice, M.L. (2002) Stability of behavioral ratings of children with specific language impairment. J Speech Lang Hear R, 45, 190-201.

19. Rice, M.L.; Haney, K.R. \& Wexler, K. (1998) Family histories of children with specific language impairment who show extended optional infinitives. J Speech Lang Hear $R, 41,419-432$.

20. Rice, M.L.; Redmond, S.M. \& Hoffman, L. (2006) MLU in children with specific language impairment and younger control children shows concurrent validity, stable and parallel growth trajectories. J Speech Lang Hear $R, 49,793-808$.

21. Rice, M.L.; Wexler, K. \& Redmond, S.M. (1999) Grammaticality judgments of an extended optional infinitive grammar: Evidence from English-speaking children with specific language impairment. $J$ Speech Lang Hear R, 42, 943-961.

22. Oetting, J.B.; Rice, M.L. \& Swank, L.K. (1995) Quick incidental learning (QUIL) of words by schoolaged children with and without specific language impairment. J Speech Hear Res, 38, 434-445.

23. Rice, M.L.; Wexler, R.; Marquis, J. \& Hershberger, S. (2000) Acquisition of irregular past tense by children with specific language impairment. J Speech Lang Hear $R, 43,1126-1145$.

24. Van der Lely, H.K.J.; Rosen, S. \& McClelland, A. (1998) Evidence for a grammar specific deficit in children. Curr Biol, 8(23), 1253-1258.

25. Van der Lely, H.K.J. \& Stollwerck, L. (1996) A grammatical specific language impairment in children: An autosomal dominant inheritance? Brain Lang, 52, 484-504.

26. Van der Lely, H.K.J. (1994) Canonical linking rules: Forward $\mathrm{v} / \mathrm{s}$ reverse linking in normally developing and specifically language impaired children. Cognition, 51, 29-72.
27. Van der Lely, H.K.J. (1997) Language and cognitive development in a grammatical specific language impairment boy: Modularity and innateness. $J$ Neurolinguist, 10, 75-107.

28. Van der Lely, H.K.J. (1998) Specific language impairment in children: Movement, economy and deficits in the computational-syntactic system. Lang Acquis, 7, 161-192.

29. Van der Lely, H.K.J. \& Stollwerck, L. (1997) Binding theory and specifically language impaired children. Cognition, 62, 245-290.

30. Fodor, J. (1983) The Modularity of Mind. Cambridge: MIT Press

31. Chomsky, N. (1986) Knowledge of language: its nature, origins and use. New York: Praeger.

32. Pinker, S. (1994) The language instinct: How the mind creates language. New York: W. Morrow.

33. Pinker, S. (1999) Words and Rules. New York: Basic Books.

34. Van der Lely, H.K.J. \& Ullman, M. (2001) Past tense morphology in specifically language impaired children and normally developing children. Lang Cognitive Proc, $16,177-217$.

35. Johnston, J. \& Kamhi, A. (1984) The same can be less: Syntactic and semantic aspects of the utterances of language impaired children. Merrill-Palmer Quarterly, 30, 65-86.

36. Bliss, L. (1989) Selected syntactic usage of languageimpaired children. J Commun Disord, 22, 277-289.

37. Leonard, L.; McGregor, K. \& Allen, G. (1992) Grammatical morphology and speech perception in children with specific language impairment. $J$ Speech Hear Res, 35, 1076-1085.

38. Leonard, L.B. (1995) Functional categories in the grammars of children with specific language impairments.J Speech Hear Res, 38, 1270-1283.

39. Leonard, L.B.; Eyer, J.; Bedore, L. \& Grela, B. (1997) Three accounts of the grammatical morpheme difficulties of English-speaking children with specific language impairment.J Speech Lang Hear R, 40, 741-753.

40. Oetting, J.B. \& Horohov, J.E. (1997) Past-tense marking by children with and without specific language impairment. J Speech Lang Hear R, 40, 62-74.

41. Bishop, D. (1992) The underlying nature of specific language impairment. J Child Psychol Psyc, 33, 3-66. 
42. Gillam, R.B.; Cowan, N. \& Marler, J. (1998) Information processing by school-age children with specific language impairment: Evidence from a modality effect paradigm. J Speech Lang Hear R, 41, 913-926.

43. Roediger, H.L. (1980) Memory metaphors in cognitive psychology. Mem Cognition, 8, 231-246.

44. Salthouse, T.A. (1985) A Theory of Cognitive Aging, North-Holland, Amsterdam (pp. 18-42).

45. Kail, R. \& Salthouse, T. A. (1994) Processing speed as a mental capacity. Acta Psychol, 86, 199-225.

46. Gillam, R.B. \& Hoffman, L.M. (2003) Information processing in children with specific language impairment. In L. Verhoeven \& H. Van Balkom (eds.), Classification of Developmental Language Disorders: Theoretical Issues and Clinical Implications. Mahwah, NJ: Lawrence Erlbaum, pp. 137-157.

47. Kail, R.; Hale, C.A.; Leonard, L.B. \& Nippold, M.A. (1984) Lexical storage and retrieval in language impaired children. Appl Psycholinguist, 5, 37-49.

48. Kirchner, D. \& Klatsky, R. (1985) Verbal rehearsal and memory in language disordered children.J Speech Hear Res, 28, 556-564.

49. Kail, R. \& Leonard, L. (1986) Word-finding abilities in language-impaired children. ASHA Monographs, 25.

50. Johnston, J.R.; Smith, L.B. \& Box, P. (1997) Cognition and communication: referential strategies used by preschoolers with specific language impairment. Journal of Speech and Hearing Sciences, 40, 964-974.

51. Johnston, J.R. \& Smith, L.B. (1989) Dimensional thinking in language impaired children. Journal of the Speech and Hearing Society, 32, 33-38.

52. Kamhi, A. (1981) Nonlinguistic symbolic and conceptual abilities of language impaired and normally developing children. J Speech Hear Res, 24, 446-453.

53. Johnston, J. \& Ramstad, V. (1983) Cognitive development in pre-adolescent language impaired children. Brit J Disord Commun, 18, 49-55.

54. Kamhi, A.; Catts, H.; Koenig, L. \& Lewis, B. (1984) Hypothesis testing and nonlinguistic symbolic abilities in language impaired children.J Speech Hear Disord, 49, 169-176.

55. Montgomery, J. (1993) Haptic recognition of children with specific language impairment: Effects of response modality.J Speech Hear Res, 36, 98-104.
56. Ellis-Weismer, S. (1985) Constructive comprehension abilities exhibited by language impaired children. $J$ Speech Hear Res, 28, 175-184.

57. Bishop, D.V.M. \& Adams, C. (1992) Comprehension problems in children with specific language impairment: Literal and inferential meaning. J Speech Hear Res, 35, 119-129.

58. Hayiou-Thomas, M.E.; Bishop, D.V.M. \& Plunkett, K. (2004) Simulating specific language impairment: general cognitive processing stressors can produce a specific linguistic profile. J Speech Lang Hear R, 47, 13471362.

59. Johnston, J. (1991) Questions about cognition in children with specific language impairment. In J. Miller (eds.) Research on child language disorders. Austin: Pro-Ed.

60. Johnston, J. (1994) Cognitive abilities of children with language impairment. In R. Watkins \& M. Rice (eds.) Specific Language Impairment in Children. Baltimore: Paul H. Brookes, pp 107-121.

61. Leonard, L. (1989) Language learn ability and specific language impairment in children. Appl Psycholinguist, 10, 179-202.

62. Montgomery, J. \& Leonard, L. (1998) Real-time inflectional processing by children with specific language impairment: Effects of phonetic substance.J Speech Lang Hear $R, 41,1432-1443$.

63. Van der Lely, H. (1996) Language modularity and grammatical specific language impairment. In M. Aldridge (eds.) Child language. Clevedon, U.K.: Multilingual Matters, pp. 188-201.

64. Bishop, D.V.M. (1989) Test for the Reception Of Grammar (TROG). Manchester: University of Manchester.

65. Leonard, L. \& Bortolini, U. (1998) Grammatical morphology and the role of weak syllables in the speech of Italian-speaking children with specific language impairment. J Speech Lang Hear R, 41, 1363-1374.

66. Leonard, L. (1992) The use of morphology by children with specific language impairment: Evidence from three languages. In R. Chapman (eds.) Processes in language acquisition and disorders. Chicago: Mosby-Yearbook, pp. 186-201.

67. Dromi, E.; Leonard, L. \& Shteiman, M. (1993) The grammatical morphology of Hebrew-speaking children with specific language impairment: Some competing hypotheses. J Speech Hear Res, 36, 760-771. 
68. Leonard, L.; Bortolini, U.; Caselli, M.C. \& Sabbadini, L. (1993) The use of articles by Italianspeaking children with specific language impairment. Clin Linguist Phonet, 7, 19-27.

69. Bortolini, U.; Caselli, M.C. \& Leonard, L. (1997) Grammatical deficits in Italian-speaking children with specific language impairment. J Speech Hear Res, 40, 809820.

70. Dromi, E.; Leonard, L.; Adam, G. \& ZadunaiskyEhrlich, S. (1999) Verb agreement morphology in Hebrew-speaking children with specific language impairment. J Speech Lang Hear R, 42, 1414-1431.

71. Bedore, L. \& Leonard, L. (2000) The effects of inflectional variation on fast mapping of verbs in English and Spanish.J Speech Lang Hear R, 43, 21-33.

72. Bishop D.V.M. \& Leonard, L.B. (2000) Speech and language impairments in children: Causes, characteristics, intervention and outcomes. East Sussex: Psychology Press.

73. Tallal, P. \& Piercy, M. (1973) Defects of non-verbal auditory perception in children with developmental aphasia. Nature, 241, 468-469.

74. Tallal, P. (1980) Auditory temporal perception, phonics and reading disabilities in children. Brain Lang, 9, 182198.

75. Merzenich, M.M., Jenkins, W.M.; Johnston, P.; Schreiner, C.; Miller, S.L. \& Tallal, P. (1996) Temporal processing deficits of language-learning impaired children ameliorated by training. Science, 271, 77-81.

76. Tallal, P. (2000) Experimental studies of language learning impairments: From research to remediation. In Bishop, D.V.M. \& L.B. Leonard (eds.) Speech and language impairments in children: Causes, characteristics, intervention and outcome. Hove, U.K.: Psychology Press, pp. 131-155.

77. Burlingame, I.; Sussman, H.; Gillam, R.B. \& Hay, J. (2005) An investigation of speech perception in children with specific langauge impairment on a continuum of formant transition duration. J Speech Lang Hear R, 48 (4), 805-816.
78. Corriveau, K.; Pasquini, E. \& Goswami, U. (2007) Basic auditory processing skills and specific langauge impairment: A new look at an old hypothesis. J Speech Lang Hear R, 50, 647-666.

79. Ziegler, J.C.; Pech-George, C.; George, F; Alario, F.X. \& Lorenzi, C. (2005) Deficits in speech perception predict language learning impairment. Proceedings of the National Academy of Science, 102(141), 10-15.

80. Gathercole, S. \& Baddeley, A. (1990) Phonological memory deficits in language-disordered children: Is there a causal connection? J Mem Lang, 29, 336-360.

81. Gathercole, S. \& Baddeley, A. (1993) Working Memory and Language. Hove, Sussex: Lawrence Erlbaum Associates.

82. Sussman, J. (1993) Perception of formant transition cues to place of articulation in children with language impairments. J Speech Hear Res, 36, 1286-1299.

83. Gillam, R.B.; Cowan, N. \& Day, L. (1995) Sequential memory in children with and without language impairment. J Speech Hear Res, 38, 393-402.

84. Montgomery, J.W. (1995) Examination of phonological working memory in specifically language impaired children. Appl Psycholinguist, 16, 335-378.

85. Gillam, R.B. (2002) The Role of Working Memory in Vocabulary Development. Perspectives on Language. Learning, and Education, 6, 3-5.

86. Gillam, R.B.; Hoffman, L.M.; Marler, J.A. \& Wynn-Dancy, M.L. (2002) Sensitivity to increased task demands: Contributions from data-driven and conceptually driven processing deficits. Top Lang Disord, 22 (3), 30-49.

87. Baddeley, A. (1986) Working memory. Oxford: Clarendon.

88. Hoffman, L.M. \& Gillam, R.B. (2004) Verbal and spatial information processing constraints in children with specific language impairment. J Speech Lang Hear $R, 47,114-125$.

89. Ullman, M.T. \& Pierpont, E.I. (2005) Specific language impairment is not specific to language: The procedural deficit hypothesis. Cortex, 41(3), 399-433. 